\title{
Color management of semi-transparent nano-patterned surfaces
}

\author{
Fabien Mandorlo $\odot,{ }^{\mathrm{a}, *}$ Mohamed Amara, ${ }^{\mathrm{a}}$ Hai Son Nguyen, ${ }^{\mathrm{b}}$ \\ Arthur Charlety-Meano, ${ }^{a}$ Ali Belarouci, ${ }^{b}$ and Régis Orobtchouk $\odot^{a}$ \\ ${ }^{a}$ Université de Lyon, Institut des Nanotechnologies de Lyon INL-UMR5270 CNRS, \\ INSA Lyon, Villeurbanne, France \\ ${ }^{b}$ Ecole Centrale de Lyon, Institut des Nanotechnologies de Lyon INL-UMR5270 CNRS, \\ INSA Lyon, Villeurbanne, France
}

\begin{abstract}
Many applications require colored surfaces while maximizing photons to pass through with no optical losses. As the perceived color depends on the spectral content and the number of reflected photons for each wavelength, it is necessary to consider not only the chromatic content but also the brightness. As a first application example, photovoltaic modules could see their acceptance in urban situations greatly improved if their color could be controlled while maximizing the number of photons used for energy conversion. In this case, it is important to limit light brightness to avoid glare when light coming from the sun is reflected directly. As another example, a colored and semi-transparent glass layer in the infrared could efficiently protect sensors while camouflaging them, such as lidar in automotive applications. We propose a theoretical study for multicolor applications, considering 2D partially etched grating waveguide structures that behave as a perfect broadband antireflection coating while the residual layer couples light in a waveguided mode in order to reflect only a particular photon band. This strategy allows to produce a large variety of colors and the use of non-absorbing materials avoids any optical loss. The width of the resonance peak is a crucial parameter for balancing brightness in multicolor applications. In the second part, we developed a method for a fast optimization of the geometrical parameters and we applied it to obtain the three primary colors with strong geometrical constraints, so that such structures could be manufactured in a single step by nanoimprint. Finally, we propose some improvements of these structures, in particular by reducing broadband reflection to obtain more saturated colors, or to improve the angular behavior of these structures. () 2021 Society of Photo-Optical Instrumentation Engineers (SPIE) [DOI: 10.1117/1.OE.60.5 $.055101]$
\end{abstract}

Keywords: CIE 1931 color space; photonic crystal; patterning; sRGB; brightness.

Paper 20201152 received Sep. 28, 2020; accepted for publication Apr. 6, 2021; published online May 13, 2021.

\section{Introduction}

Grating waveguide structures (GWSs) have been considered this last decade to obtain tiny resonant bandpass filters that can be used to control high chromaticity in the color printing field as shown in Refs. 1-3. In the indoor photovoltaic area, for example, merging elementary pixels based on GWS with different periods on top of the front glass would lead to the fabrication of colored panels without a strong degradation of the conversion efficiency. Performance items required for such filters are a broadband antireflection coating coupled to a sufficient narrow reflection resonance involving accurate perception of color, with limited color variations depending on the optical conditions as described in Ref. 4.

In this paper, we focus on a GWS that behaves both as a bandpass color filter and as a single layer anti-reflection coating (s-ARC). We will first assume that its reflected spectrum has only one peak which can be defined by its central wavelength, its width, and the level of background reflection. These three independent parameters impact the colors perceived by the human eye,

*Address all correspondence to Fabien Mandorlo, fabien.mandorlo@ INSA-Lyon.fr

0091-3286/2021/\$28.00 (C) 2021 SPIE

Optical Engineering

055101-1

May $2021 \cdot$ Vol. 60(5) 
such as brightness, saturation, and hue. In the first part, we carry out a theoretical study of these parameters in order to predict the overall shape of the reflected spectrum to produce a desired color. For multicolored applications, this part also aims to highlight the relationships to be considered between the different reflected spectra. In the second part, we focus on GWS structures and their simulation methodology and optimization to control the reflected spectrum and so the reflected color.

\section{Range of Colors Accessible by Patterning the Front Glass Layer}

To reduce the number of parameters to study an ARC on a glass substrate, we consider that the reflected spectrum has a Lorentzian shape centered on wavelength $\lambda_{c}$, with a width to mid height $\Delta \lambda_{c}$. In the case of a resonant mode, the maximum of the reflection is equal to 1 , but a reflection on the entire visible spectrum $R_{0}$ will eventually be taken into account, leading to an amplitude of the reflected signal equal to $1-R_{0}$,

$$
R(\lambda)=R_{0}+\frac{1-R_{0}}{1+\left[\frac{2 \cdot\left(\lambda-\lambda_{c}\right)}{\Delta \lambda_{c}}\right]^{2}} .
$$

These assumptions restrict the study to only three parameters: $\lambda_{c}, \Delta \lambda_{c}$, and $R_{0}$. Using this theoretical expression of $R(\lambda)$, it is possible to calculate the CIE (International Commission on Illumination) $1931 x y Y$ components using the three color matching functions $\bar{x}, \bar{y}$, and $\bar{z}$ as explained in Ref. 4. However, this color space is not perceptually uniform. For this reason, we prefer Judd's Uniform Chromaticity Scale (UCS) diagram using Ref. 5,

$$
\left\{\begin{array}{l}
u=\frac{0.4661 \cdot x+0.1593 \cdot y}{y-0.15735 \cdot x+0.2424} \\
v=\frac{0.6581 \cdot y}{y-0.15735 \cdot x+0.2424}
\end{array} .\right.
$$

As the $(u, v, Y)$ diagram is uniform, we can use the Euclidian distance between two colors $C_{1}\left(u_{1}, v_{1}, Y_{1}\right)$ and $C_{2}\left(u_{2}, v_{2}, Y_{2}\right)$ and calculate gamut coverage areas. Unlike other color spaces (CIE $1964 U^{*} V^{*} W^{*}$, CIE $1976 L^{*} a^{*} b^{*}$, or CIE $1976 L^{*} u^{*} v^{*}$ ), it does not depend on a reference illuminant for its white point (WP): most often, it corresponds to daylight at temperature $6504 \mathrm{~K}$ (D65) or 5003K (D50) and color coordinates are relative to the chosen WP. In addition, the chromaticity is contained in a 2D plane (as for the CIE $1931 x y Y$, CIE $1964 U^{*} V^{*} W^{*}$, CIE $1976 L^{*} u^{*} v^{*}$, and contrary to CIE $1976 L^{*} a^{*} b^{*}$ and its derivated color spaces), which makes color representation easier when $Y$ stands for the corresponding brightness. Finally, we chose the model derived from Judd's work from Ref. 5 that has been historically less exploited than the simplified (for computational purposes) version of MacAdam proposed in 1937 in Ref. 6, which was adopted by CIE in 1960 .

Finally, in this article, we consider that the reference spectrum $I_{0}$ is AM1.5D which represents the light emitted directly by the sun for an air mass of 1.5. As a consequence, the calculated colors correspond to an outdoor use and the resulting WP corresponds to $R_{0}=1$ at coordinates $(u=0.3966, v=0.4273)$.

\subsection{Study of an Ideal Optical Response}

In this part, we consider the ideal case $R_{0}=0$ in Eq. (1) in order to determine the optical properties for a very favorable case and for a single resonant mode. In that case, the calculated brightness is called $Y_{0}$.

Figure 1 represents the chromaticity when the position $\lambda_{c}$ of the Lorentzian varies for different values of $\Delta \lambda_{c}$. For each value of $\Delta \lambda_{c}$, the gamut starts toward the WP: when $\lambda_{c}$ increases, the calculated color approaches the blue color and sweeps the rainbow colors in direction of the reddest point. Then, the curve goes back to the WP again.

Unsurprisingly, the gamut is the most extended for a narrow resonance $\left(\Delta \lambda_{c}=1 \mathrm{~nm}\right)$ and it gets closer to WP when the width of the peaks $\Delta \lambda_{c}$ increases. This behavior is a direct 


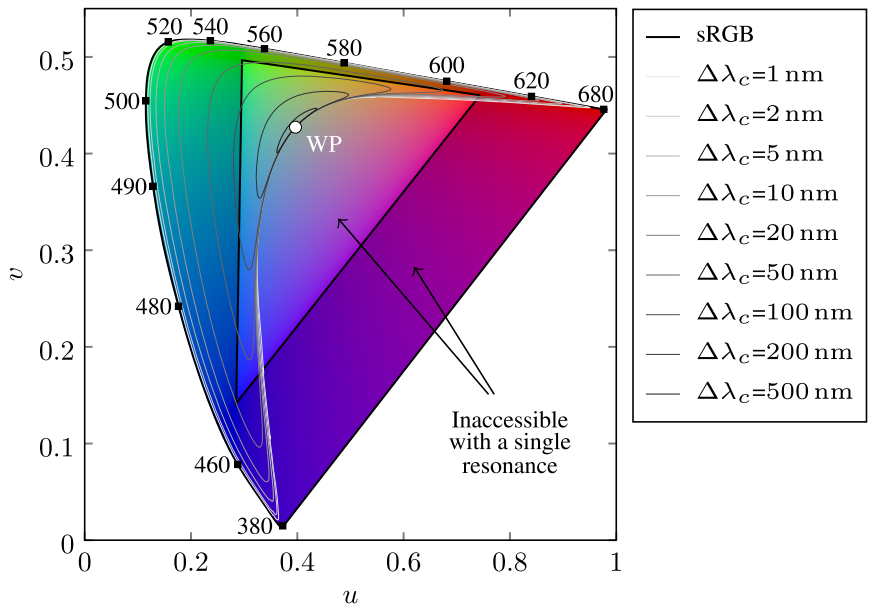

Fig. 1 Different gamuts for an ideal case with no constant reflection in the visible range $\left(R_{0}=0\right)$. Each gray contour line is obtained when varying the peak position $\lambda_{c}$ for a given value of $\Delta \lambda_{c}$. The standard red-green-blue (sRGB) domain corresponds to the colors that can be produced by monitors.

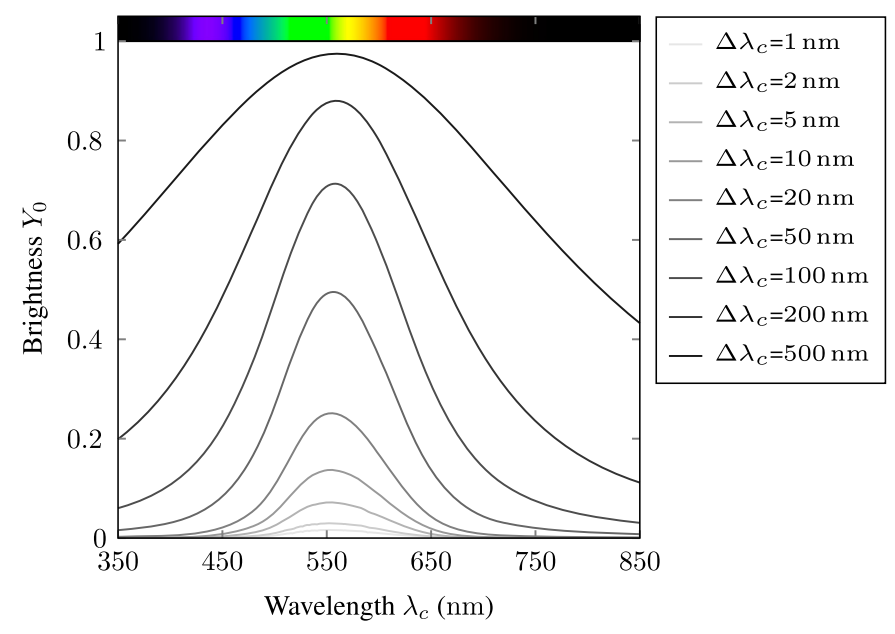

Fig. 2 Color brightness $Y_{0}$ for the same Lorentzian functions as Fig. 1. On top of the figure, hue is given for illustration purpose.

consequence of a lower spectral selectivity of the reflected light, with a decrease of the saturation of the obtained color. A maximum coverage of $39 \%$ is obtained for the thinner peak. Last, it can be observed that the area above the purple line, containing shades of blue, magenta, and red colors, cannot be obtained even if it represents $61 \%$ of the gamut surface: for these colors, a photonic crystal (PC) with two simultaneously reflected peaks is necessary.

For each curve from Fig. 1, brightness $Y_{0}$ varies significantly with an envelope similar to that of the function $\bar{y}$ (as plotted in Ref. 4), as shown in Fig. 2. As the human eye is very sensitive to the green color, the width of the PC resonance must be increased when moving away from the green color. To obtain $Y_{0}=1$, every photon in the visible range must be reflected, leading to $\Delta \lambda_{c}=+\infty$ and corresponding to the WP in the gamut.

For an arbitrary target brightness $Y_{0}=0.4, \Delta \lambda_{c}$ in the 20- to 50-nm range is enough for the green color while it is necessary to consider wider than 200-nm peaks to balance with red and blue colors. According to Fig. 1, this brings the perceived color very close to the WP, with a poor saturation. On the other hand, we notice a relative symmetry around the wavelength corresponding to the green color $(560 \mathrm{~nm})$ : to balance the brightness of a blue $(473 \mathrm{~nm})$ and a red surface $(650 \mathrm{~nm})$, we need $\Delta \lambda_{c}$ between 100 and $150 \mathrm{~nm}$. 


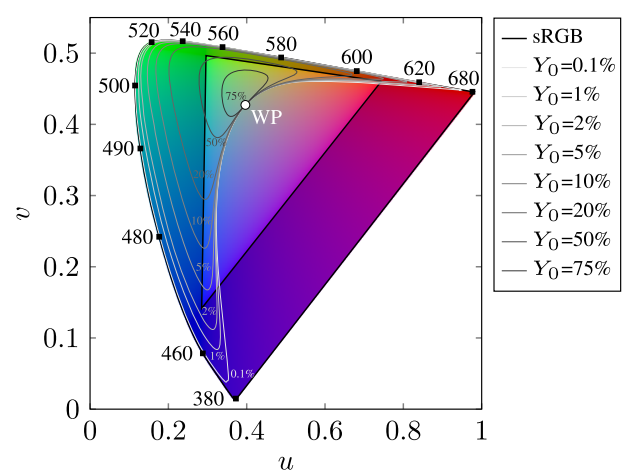

(a)

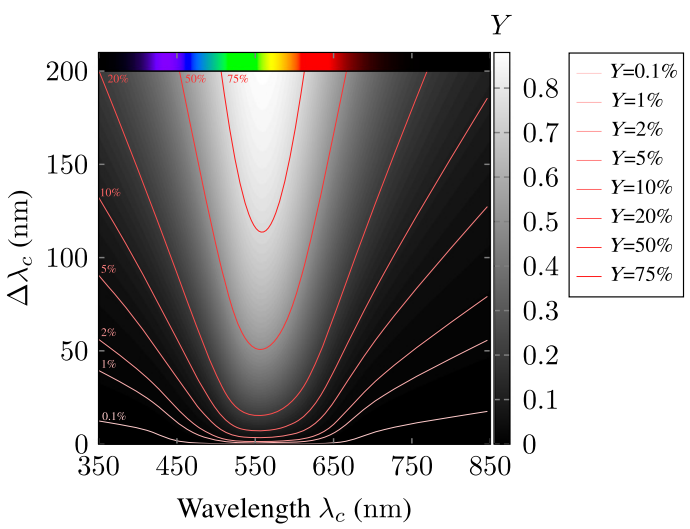

(b)

Fig. 3 Iso luminance curves for an ideal case with no constant reflection in the visible range $\left(R_{0}=0\right)$. (a) Chromaticity diagram of with few iso luminance curves and (b) relation between the wanted color $\left(\lambda_{c}\right)$ and the width of the reflected signal $\left(\Delta \lambda_{c}\right)$. On top of the figure, hue is given for illustration purpose.

\subsection{Maximum Brightness for a Single Mode PC}

For multicolor applications, we may want to control the brightness of each color. For this purpose, we determined in Fig. 3(a) the gamut obtained for different constant values of the brightness $Y_{0}$. This curve is very close to that obtained in Ref. 5 (Fig. 13, in the CIE $1931 x y Y$ diagram) which gives curves of constant excitation purity, except in the bottom part of the gamut. The more we look for a significant brightness, the closer we get close to the WP by mainly reducing the extension of the achievable colors in the red and blue directions. However, if such a structure is directly illuminated by the sun, it is important not to dazzle humans. It is consequently advised not to exceed the ambient brightness, which corresponds to approximately $Y_{0}=10 \%$ when we consider the diffuse level contained in the AM1.5G spectrum and AM1.5D as illuminant. The curve $Y_{0}=10 \%$ shows that we can therefore theoretically reach and exceed the green and red colors of the sRGB domain, and come very close to the blue color.

Figure 3(b) represents the relationship between $\lambda_{c}$ and $\Delta \lambda_{c}$ for the same $Y$ constants as in Fig. 3(a). For wavelengths below $500 \mathrm{~nm}$ and greater than $650 \mathrm{~nm}$, these two parameters vary almost linearly with each other. One may notice that when $Y_{0}$ increases, the blue and red colors become more and more difficult to obtain: for $Y_{0}=0.75$, shades of red, orange, yellow, and green can only be obtained for less than 200-nm-width peaks that cannot be currently fabricated with a PC since it exploits resonances. On the contrary, for very contrasting colors, a low brightness allows to get all the colors of the rainbow with a narrow single resonance. Last, this figure can easily be used as an abacus to obtain the stencils of the reflection spectra of each wanted color of a polychromatic drawing, each consisting of a PC for instance.

\subsection{Impact of a Residual Reflection}

In this part, we focus on the impact of $R_{0}>0$ since this constant represents a reflection on the whole visible spectrum. It can be the consequence of

- a patterned layer that does not behave as an efficient anti-reflective coating around the resonance;

- a parasitic reflection under the glass substrate (Fig. 4). For instance, considering the front glass layer constituting the encapsulation of a solar cell in its module, this constant allows to take into account the reflected photons on the solar cell which escape from the front surface of the module.

Since the brightness calculation is a scalar product (as detailed in Ref. 4), its linearity implies that the brightness $Y_{R_{0}}$ can be expressed according to that $\left(Y_{0}\right)$ when $R_{0}=0$ (previous paragraph), 


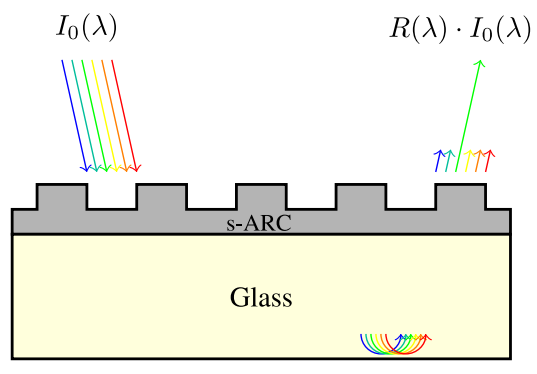

Backside reflection

Fig. 4 Schematic structure considering a backside reflection and a green dominating peak for the reflection.

$$
Y_{R_{0}}=R_{0}+\left(1-R_{0}\right) \cdot Y_{0}>R_{0}
$$

For small values of $R_{0}$, this equation is approximated by

$$
Y_{R_{0}} \approx R_{0}+Y_{0} \text {. }
$$

This approximation shows that the abacus in Fig. 3(b) can be used to calculate the brightness $Y_{R_{0}}$ when $R_{0} \neq 0$. For example, for $R_{0}=2 \%$, just use the level curve $Y_{0}=3 \%$ to get a brightness $Y_{R_{0}}$ of $5 \%$ and adjust the wanted peaks properties (widths and respective positions).

In addition to impacting the color brightness, $R_{0}$ significantly modifies the obtained gamuts. As can be seen in Fig. 5 for $R_{0}=1 \%$ and $R_{0}=2 \%$, when $Y_{R_{0}}$ is close to $R_{0}$, the gamut is extremely small. Indeed, the photons responsible for the desired color are dominated by the number of photons emitted over the entire visible spectrum. For very low brightness $\left(Y_{R_{0}} \sim R_{0}\right)$, the most saturated colors correspond to blue ones, since the $\bar{x}$ color matching function (plotted in Ref. 4) is narrower than the $\bar{y}$ and $\bar{z}$ ones. Last, when the total brightness $Y_{R_{0}}$ increases and dominates $R_{0}$, the gamut reaches a maximum area (around $Y_{R_{0}}=10 \%$ for $R_{0}=1 \%$ and $Y_{R_{0}}=20 \%$ for $R_{0}=2 \%$ ) before shrinking again. This behavior can be explained by the Center of Gravity Law of color mixture (see Sec. 2.4) since adding a constant reflection leads to a resulting color closer to the WP. Last, the dashed line between the "bluest" point, WP, and the reddest one delimits the border of the colors that can be achieved; this surface is smaller than with $R_{0}=0$ (Fig. 1) since it corresponds to $49.8 \%$ of the gamut (instead of $61 \%)$.

\subsection{Taking the Most of Multimode PC}

In the previous section, we have shown that for a given resonance, we obtain a color $C$ in the form of a triplet $(x, y, Y)$ or $(u, v, Y)$ which determines the maximum brightness $Y=Y_{\max }$ accessible with a single resonant mode. To obtain a color $C^{\prime}$ of identical chromaticity $(x, y)$ with an intermediate brightness $Y$ in range $\left[0, Y_{\max }\right]$, we can mix $n$ resonances giving colors from $C_{1}$ to $C_{n}$. Because of the Center of Gravity Law of color mixture detailed in Ref. 7, the resulting color $C_{r}$ is a new triplet $\left(x_{r}, y_{r}, Y_{r}\right)$ defined as follows:

$$
x_{r}=\frac{\sum_{i=1}^{n} \frac{x_{i} \cdot Y_{i}}{y_{i}}}{\sum_{i=1}^{n} \frac{Y_{i}}{y_{i}}}, \quad y_{r}=\frac{\sum_{i=1}^{n} Y_{i}}{\sum_{i=1}^{n} \frac{Y_{i}}{y_{i}}}, \quad Y_{r}=\sum_{i=1}^{n} Y_{i} .
$$

In the case of two resonant modes giving colors $C_{1}$ and $C_{2}$, the resultant chromaticity $\left(x_{r}, y_{r}\right)$ is on the segment connecting those of $C_{1}$ and $C_{2}$ both in the CIE $1931 x y Y$ and the UCS color spaces. Thus, by taking two photonic resonances, centered at $\lambda_{c_{1}}$ and $\lambda_{c_{2}}$ of width $\Delta \lambda_{c_{1}}$ and $\Delta \lambda_{c_{2}}$, one can obtain a color in the central part of the gamut, with a controlled low brightness if each peak is very narrow (low values of $\Delta \lambda_{c_{1}}$ and $\Delta \lambda_{c_{2}}$ ). This property also makes it possible to obtain 


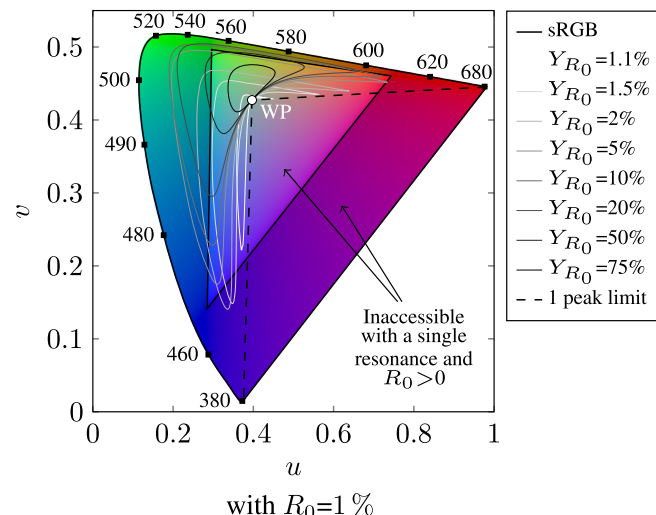

(a)

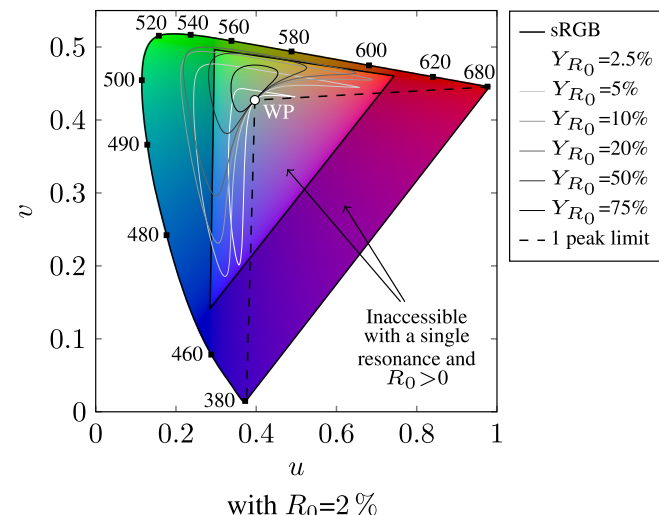

(b)

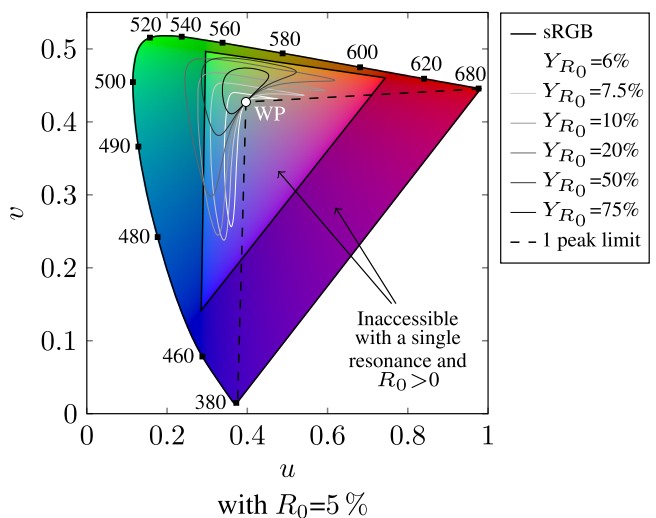

(c)

Fig. 5 Iso luminance curves for a constant reflection in the visible range: (a) with $R_{0}=1 \%$, (b) with $R_{0}=2 \%$, and (c) with $R_{0}=5 \%$.

colors in the blue-magenta-red area which is inaccessible with a single resonance. However, this means that we need PCs

- with a single resonance for the brightest or purest colors and

- with two (or more) resonances to access additional colors or to adjust the brightness to the wanted level.

In both cases, for a correct color, whatever the position of the observer, it is important that these modes are not (or very weakly) sensitive to the angle of incidence $\theta_{0}$.

\section{Simulation Methodology to Size a s-ARC Using a 2D Photonic Crystal}

\subsection{Description of the Structure}

In this part, we focus on the design of a s-ARC where light at normal incidence will be almost totally transmitted through the patterned layer, except for few wavelengths. Such a PC can be fabricated using a square array of cylindrical patterns, where the filling fractions $(f f)$ represent the amount of surface that is not etched while patterning. For a single etched layer (or a stack of different dielectric layers), the main parameters are:

- the dispersion of each considered material. To avoid optical losses, none of the considered materials should be absorbing in the visible range;

- the thickness of the patterned layer $t_{\mathrm{ARC}}$;

- the $f f$ of the PC, equal to $\pi \cdot(r / P)^{2}$ for pillars and $1-\pi \cdot(r / P)^{2}$ for holes; and

- the period $P$ of the PC. 


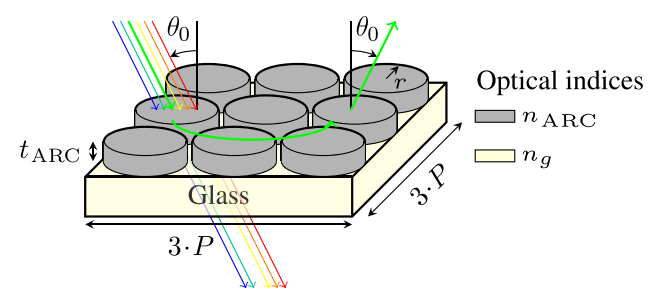

Fig. 6 Example of a s-ARC reflecting the green color in case of a 2D array of pillars on glass made of a single dielectric layer with optical index $n_{\text {ARC }}$.

Figure 6 shows an illustration when a single dielectric layer $\left(n_{\text {ARC }}\right)$ is patterned to obtain a $2 \mathrm{D}$ array allowing the green color to be reflected. This $2 \mathrm{D}$ array behaves as a bandpass filter for the reflected light if two conditions are satisfied.

1. Out of the resonant wavelength, the filter acts as a broadband ARC due to the Fabry-Perot cavity (vertical direction) leading to destructive interferences for the reflected light. To make a link with the previous part, a more realistic description of $R_{0}(\lambda)$ should be the optical response of this filter as illustrated in Ref. 8.

2. A sharp resonance of the reflection and transmission occurs due to the destructive interference between the specular transmission beam and the waveguided mode localized in the 2D periodic array. This effect, called a Fano resonance, produces a peak on the reflection spectrum at position $\lambda_{c}$. In the previous part, this peak has been modeled by a Lorentzian to control its shape with only two parameters instead of three.

From now, we propose to study the structure of Fig. 6 in two steps that can be calculated easily.

1. Choose the appropriate material for the s-ARC $n_{\mathrm{ARC}}$ and determine the thickness $t_{\mathrm{ARC}}$ of this layer and its filling factor $f f$.

2. Consider the wanted color and determine the right value of the period $P$.

\subsection{Fabry-Perot Behavior in the Visible Range}

In this part, we assume that the device is not encapsulated, so the ambient media is air, with an optical index $n_{0}=1$. As a wide range with a flat and low residual reflection is necessary, we focus on a single layer ARC. To easily size the structure, we define a linear equivalent optical index for the s-ARC layer (Fig. 7),

$$
\left(n_{\mathrm{ARC}}^{\mathrm{eq}}\right)^{2}=n_{\mathrm{ARC}}^{2} \cdot f f+(1-f f) \cdot n_{0}^{2} .
$$

The $f f$ can be adjusted so this optical index also satisfies the following equation to minimize the reflection over a wide range of wavelengths:

$$
n_{\mathrm{ARC}}^{\mathrm{eq}}=\sqrt{n_{g} \cdot n_{0}}
$$

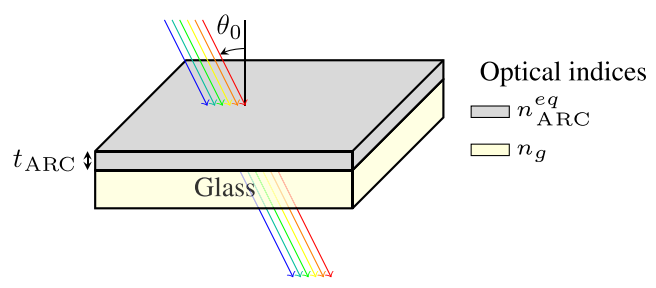

Fig. 7 Equivalent structure of Fig. 6 to size the optical behavior except at the PC resonance and get $R_{0}$ as low as possible. 
As a consequence, to conciliate both Eqs. (6) and (7), we need

$$
f f=n_{0} \cdot \frac{n_{g}-n_{0}}{n_{\mathrm{ARC}}^{2}-n_{0}^{2}} \approx \text { constant. }
$$

As $f f$ may not be constant, we suggest to weight $f f(\lambda)$ by the color matching function $\bar{y}$ since this function measures the brightness and does not depend on the considered illuminating spectrum. Finally, a single quarter-wavelength coating leads to fix the thickness $t_{\mathrm{ARC}}$ since $t_{\mathrm{ARC}}=\lambda_{0} /\left(4 \cdot n_{\mathrm{ARC}}^{\mathrm{eq}}\right)$, where $\lambda_{0}$ is chosen in the middle of the visible range. As the bandwidth of ARC is directly linked to the optical path, the study is limited to the smallest values of $t_{\mathrm{ARC}}$ providing the broaden ARC spectral range.

These values $\left(t_{\mathrm{ARC}}\right.$ and $\left.f f\right)$ are used as initial guesses of a minimization routine based on a Levenberg-Marquardt algorithm (as detailed in Ref. 9) and the rigorous coupled-wave analysis (RCWA) method (as explained in Ref. 10). For this rigorous calculation, a sub-wavelength period of $100 \mathrm{~nm}$ is used in order to remove the reflection peak resonance since we can notice that for higher periods, the ARC behavior of the filter remains the same as the perturbation induced by the Fano resonance effect occurs only on a small range of wavelength in the vicinity of the reflection peak. Consequently, at this initial step, the resonant reflection peak is not present in the optical response so a wide spacing between the calculated wavelengths is enough (for instance, only 7 points between 300 and $900 \mathrm{~nm}$ ), providing a fast optimization of these two parameters in only few minutes.

\subsection{Selection of the Reflected Wavelength}

As thickness and filling factor are now calculated, the different resonances must be tuned to the desired values that can be achieved by the geometry of the PC (pattern and period). To adjust the wanted reflected signal at wavelength $\lambda_{c}$, we need to consider the propagation in the plane of the s-ARC layer (Fig. 8). Then, light escapes on the front side with an angle $\theta_{0}$. On the glass substrate side, the emitted signal may interfere with the incident signal that has directly passed through the ARC layer.

Depending on the polarization of the propagative mode, the equivalent optical index of the central region is given at first approximation in Ref. 11,

$$
\left\{\begin{array}{ll}
\left(n_{\mathrm{ARC}}^{\mathrm{eq}}\right)^{2}=n_{\mathrm{ARC}}^{2} \cdot f f+(1-f f) \cdot n_{0}^{2}, & \text { TE polarization } \\
\frac{1}{\left(n_{\mathrm{ARC}}^{\mathrm{eq}}\right)^{2}}=\frac{f f}{n_{\mathrm{ARC}}^{2}}+\frac{1-f f}{n_{0}^{2}}, & \text { TM polarization }
\end{array} .\right.
$$

We can notice that a more accurate formulation can be used involving the grating period $P$. In this study, we use the first formulation in order to illustrate that $f$ mainly rules the effective optical indices while $P$ controls the wanted reflected wavelength $\lambda_{c}$.

Then, the effective index method for an anti-symmetric three layers slab waveguide is used (as detailed in Ref. 12) to calculate $n_{\mathrm{eff}(j)}^{\mathrm{TE}}$ and $n_{\mathrm{eff}(j)}^{\mathrm{TM}}$. Depending on $t_{\mathrm{ARC}} / \lambda_{c}$, different orders $j$ may exist. Due to the grating equation and considering an arbitrary order $m$, the Bragg equation gives

$$
n_{\mathrm{eff}(j)}^{\mathrm{TE} / \mathrm{TM}}=n_{0} \cdot \sin \left(\theta_{0}\right) \pm \frac{m \cdot \lambda_{c}}{P},
$$

where $\theta_{0}=0$ at normal incidence, and $\theta_{0}$ corresponds to the angle at which the selected signal escapes from the PC. This equation leads to a very simple relationship between the selected wavelength $\lambda_{c}$ and the period $P$ of the PC,

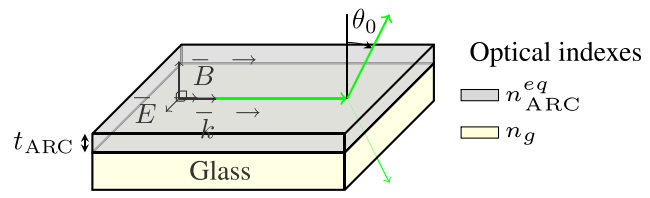

Fig. 8 Illustration of the propagation of the wanted color (TE mode) in the equivalent ARC layer. 


$$
\lambda_{c}=\frac{n_{\mathrm{eff}(j)}^{\mathrm{TE} / \mathrm{TM}} \cdot P}{k} .
$$

For a given order of the propagating mode, $n_{\mathrm{eff}(j)}^{\mathrm{TE}}>n_{\mathrm{eff}(j)}^{\mathrm{TM}}$ as demonstrated in Ref. 13. Consequently, once the period is chosen, if the system is sized for the TE mode, another color for the TM mode may create another peak at a shorter wavelength. From the analysis in the previous part, this property makes it possible to access the blue-purple-red side of the gamut depending on the chosen material and chosen geometry $\left(t_{\mathrm{ARC}}, f f, P\right)$. In addition, by sacrificing range of the low reflectivity with a thicker ARC layer, it is possible to increase the number of modes $j$ in the visible range, and thus adjust the brightness of the colors obtained.

The chosen geometry is then used as a starting point for more rigorous calculation with home-made RCWA.

\subsection{Leakage Control of the Selected Mode}

Whatever the polarization, Eq. (9) leads to $n_{\mathrm{ARC}}^{\mathrm{eq}}$ in between $n_{0}$ and $n_{g}$. Consequently, the selected colors correspond to leaky modes and part of the incident light is therefore transmitted through the substrate, reducing the reflected signal and so the color saturation. To avoid this unwanted effect, different solutions can be considered:

- partially etch (depth $t_{s}$ ) the glass substrate [Fig. 9(a)], so light is confined in the media with optical index $n_{\mathrm{ARC}}$. Here, $t_{g}$ must be high enough to avoid leakage, leading to few hundred nanometers. The partial etch of the glass layer may induce an effective index lower than the substrate itself, leading to an increase of the background reflection $R_{0}$;

- partially etch the ARC layer [Fig. 9(b)] so that a very thin $\left(t_{\text {slab }}<20 \mathrm{~nm}\right)$ layer remains with optical index $n_{\mathrm{ARC}}>n_{g}$.

In the first case, the issue may be the ARC behavior, since for all other wavelengths, the device looks like a double-layer ARC, that may reduce the transmission plateau. In the second case, the behavior of the ARC is slightly affected, and thus the study exposed in the whole of this article remains valid.

To illustrate the impact of the slab, Fig. 10 compares the reflection for different $t_{\text {ARC }}$ in the visible range with (b) and without (a) a 20-nm remaining $\mathrm{TiO}_{2}$ layer. Without the slab (a), the reflection coefficient does not exceed $1.8 \%$ for $t_{\mathrm{ARC}}=95 \mathrm{~nm}$. As the equivalent optical index is lower than the one of the glass substrate, the device does not support waveguided modes without losses giving Fano resonances with poor contrast. On the contrary, partially etching the $\mathrm{TiO}_{2}$ layer (b) results in a residual $\mathrm{TiO}_{2}$ layer of higher refractive index than the one of glass substrate. This layer reduces the losses of the waveguided mode involved in the Fano resonance. The thickness of this layer must be as low as possible to preserve the ARC properties of the structure. Calculation of the effective index of the mode with a standard optical planar waveguide mode solver as detailed in Ref. 14 shows that a residual thickness $t_{\text {slab }}$ of $20 \mathrm{~nm}$ is enough to obtain an effective index greater than the refractive index of the glass substrate $\left(n_{g}=1.46\right)$. The evolution of the reflection coefficient versus the wavelength and $t_{\mathrm{ARC}}$ with this residual layer is given in

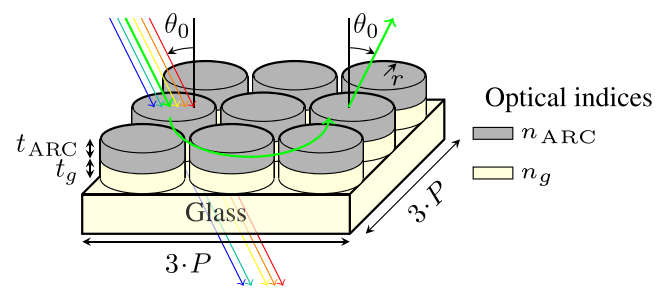

Over-etching of the glass substrate

(a)

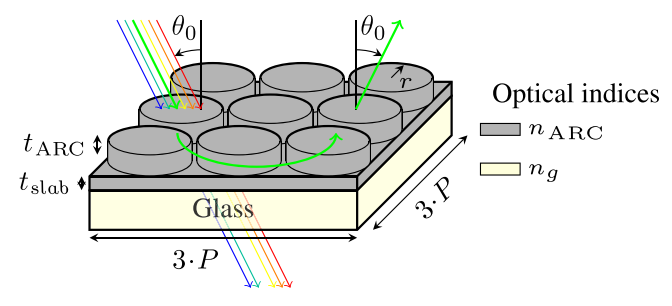

Partial etching of the ARC layer

(b)

Fig. 9 Different ways to reduce leakage for the wanted colors. (a) Overetching of the glass substrate and (b) partial etching of the ARC layer. 


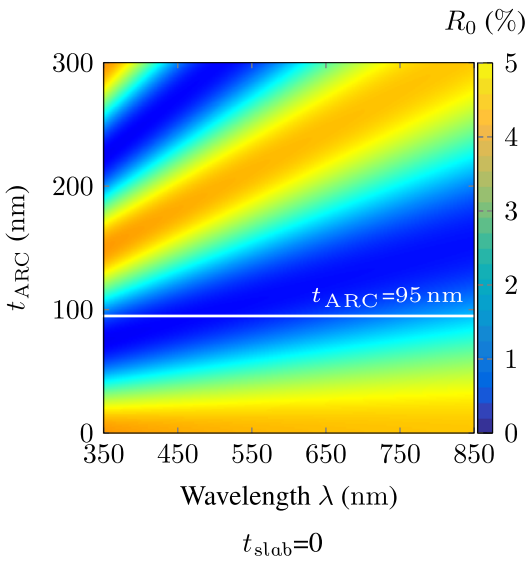

(a)

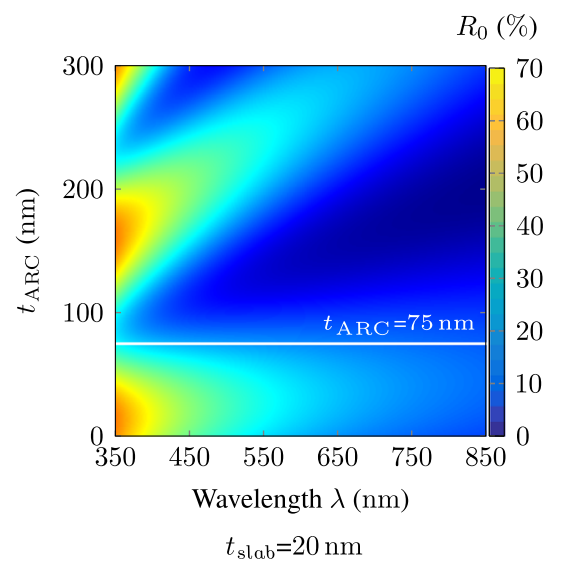

(b)

Fig. 10 Evolution of $R_{0}$ as a function of the wavelength and the thickness of the ARC for $n_{\text {ARC }}^{\text {eq }}=1.21$. (a) $t_{\text {slab }}=0$ and (b) $t_{\text {slab }}=20 \mathrm{~nm}$.

Fig. 10(b). We can notice that the reflection coefficient remains below $2.9 \%$ in the visible range for an optimized $t_{\mathrm{ARC}}$ value of $75 \mathrm{~nm}$.

\subsection{Design and Optic Optimization by RCWA}

Reflection coefficient of 2D cylindrical periodic structures is calculated with home-made RCWA code as detailed in Ref. 10. Optimization of the s-ARC follows the following procedure.

1. We calculate the optical response of the structure for sub-wavelength grating $(P=100 \mathrm{~nm})$.

2. Due to a Levenberg-Marquardt algorithm, we determine the thickness $t_{\mathrm{ARC}}$ of the remaining $\mathrm{TiO}_{2}$ layer and its filling factor $f f$ corresponding to the lowest background reflection coefficient $R_{0}$.

3. The period $P$ of the filter is set to match the Fano resonance with the wanted peak that requires the highest period. This particular choice leads to optimize the s-ARC with the biggest leakage coefficient.

4. A thin layer $t_{\text {slab }}$ on the bottom of the grating is added in a such way that the leakage of the waveguided mode matches with the coupling coefficient of the GWS (as shown in Ref. 15) giving a maximum of the reflection resonance close to 1 . At this step of the optimization process, adding $t_{\text {slab }}$ involves an increase of the background reflection coefficient $R_{0}$ : different successive iterations between steps 2 and 4 provide a trade-off between the contrast of the resonant peak and the ARC behavior of the filter. The brightness and saturation of the wanted colors are controlled at this step.

5. The period $P$ of the filter is obtained in a last step to match the Fano resonance with all the wanted wavelengths.

All the simulations are performed with refractive indexes of Tables 1 and 2.

Table 1 Experimental Sellmeier coefficients for $\mathrm{TiO}_{2}$ deposited by sputtering with a maximum error of $1.5 \%$ in the visible range.

\begin{tabular}{lccc}
\hline \hline$i$ & $A_{i}$ & $\lambda_{i}^{2}\left(\mu \mathrm{m}^{2}\right)$ & \\
\hline 1 & 3.150 & $6.922 \times 10^{-2}$ & $n(\lambda)=\sqrt{1+\sum_{i} \frac{A_{i} \cdot \lambda^{2}}{\lambda^{2}-\lambda_{i}^{2}}}$ \\
2 & 1.312 & $4.829 \times 10^{-4}$ & \\
3 & 0.607 & 155.3 & \\
\hline \hline
\end{tabular}


Table 2 Sellmeier coefficients for the amorphous $\mathrm{SiO}_{2}$ from Palik (data available in Ref. 18).

\begin{tabular}{lcc}
\hline \hline$i$ & $A_{i}$ & $\lambda_{i}^{2}\left(\mu \mathrm{m}^{2}\right)$ \\
\hline 1 & 1.103 & $8.300 \times 10^{-3}$ \\
2 & 0.364 & 43.69 \\
\hline \hline
\end{tabular}

As an example, Fig. 11 summarizes the value of the different parameters to obtain the primary colors. In this optimization process, we assume that thickness's $t_{\text {slab }}$ and $t_{\mathrm{ARC}}$ are the same for all the wanted colors: this condition would correspond to different colored surfaces fabricated in a single step, for example by a single nano-imprint process and a single mold with different patterns. This condition strongly impacts the brightness and saturation, since it impacts the width of the different peaks and the background reflection coefficient as illustrated in Fig. 12. To independently tune the brightness and saturation, one may consider more complex PCs or use a multistep process to set $t_{\text {slab }}$ and $t_{\mathrm{ARC}}$ at the best values for each wanted color.

The lowest reflection is obtained without the slab $\left(t_{\text {slab }}=0\right)$ since in the vertical direction the optical stack behaves like a perfect Bragg mirror. With the 8-nm-thick slab, reflection is higher

\begin{tabular}{|c|ccc|}
\hline Parameter & \multicolumn{3}{|c|}{ Value } \\
\hline \hline$f f(\%)$ & \multicolumn{3}{|c|}{$\leftarrow 38 \rightarrow$} \\
$t_{\text {ARC }}(\mathrm{nm})$ & \multicolumn{3}{|c|}{$\leftarrow 73 \rightarrow$} \\
$t_{\text {slab }}(\mathrm{nm})$ & \multicolumn{3}{|c|}{$\leftarrow 8 \rightarrow$} \\
$\lambda_{c}(\mathrm{~nm})$ & 650 & 530 & 470 \\
$P(\mathrm{~nm})$ & 445 & 360 & 310 \\
\hline Expected color & \multicolumn{3}{|c|}{ UCS Judd coordinates } \\
$\mathrm{u}$ & 0.4860 & 0.3383 & 0.3389 \\
$\mathrm{v}$ & 0.4356 & 0.4883 & 0.3284 \\
$\mathrm{Y}$ & 0.0802 & 0.0592 & 0.2704 \\
& sRGB approximation \\
$\mathrm{R}$ & 138 & 96 & 74 \\
$\mathrm{G}$ & 82 & 161 & 84 \\
B & 74 & 33 & 142 \\
Calculated color & \multicolumn{3}{|c}{} \\
$*$
\end{tabular}

Fig. 11 Parameters of the designed samples with a $\mathrm{TiO}_{2} \mathrm{~s}-\mathrm{ARC}$ layer.

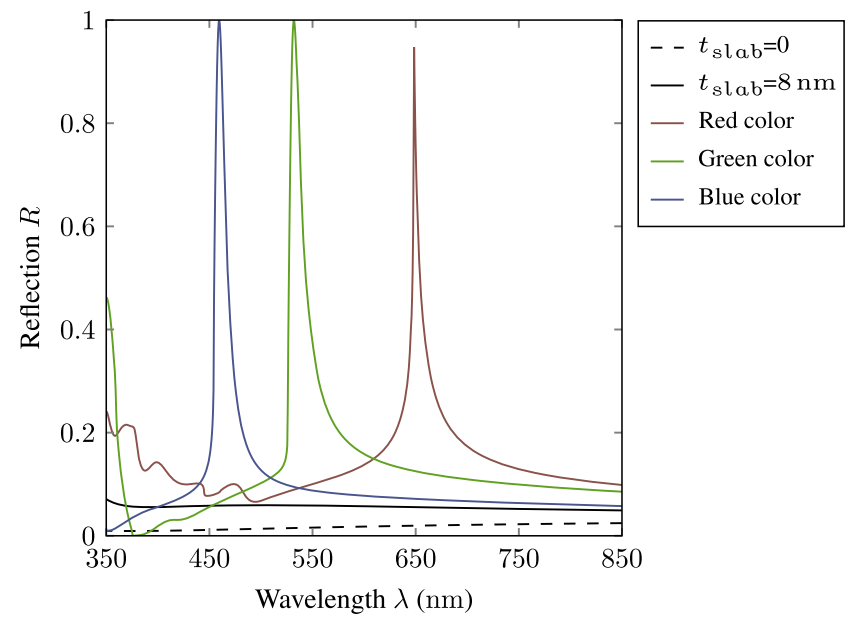

Fig. 12 Intermediate and optimized spectrum for the three primary colors. 
$\left(R_{0} \sim 5.6 \%\right)$. Then, increasing the period $P$ locates the position of $\lambda_{c}$ for each wanted color. The reflection due to the slab limits the saturation of each color (Fig. 11), and each is positioned in the sRGB triangle of the UCS Judd gamut.

This method provides the advantage of decorrelating the geometrical parameters of the grating. Furthermore, as the reflection background spectrum out of the resonance is a slowly varying curve, minimization is performed with a reduced number of points and a wavelength resolution of 50 to $100 \mathrm{~nm}$ is enough to ensure convergence of the Levenberg-Marquardt algorithm. Then, the computation time of this minimization procedure is comparable to that of the calculation of one of the curves including the Fano resonance with a resolution of $0.5 \mathrm{~nm}$.

\section{Discussion and Conclusion}

GWS make it possible to obtain structures with a very selective optical behavior. By using nonabsorbing materials, it is therefore possible to design nano-patterned layers that are capable of reflecting only certain photons at a wanted wavelength and transmitting all the others in direction of the bottom surface, which is particularly suitable to produce photovoltaic modules with a colored front glass.

The impact of the resonance properties of these GWS has been studied theoretically in the first part. If the width of the resonant peak affects both the brightness and saturation, the parasitic reflection very quickly limits the saturation of the obtained color, at the exception of blue. Despite a discontinuous solar spectrum, the width of the red and blue peaks can be almost linearly related to the chosen wavelength as soon as brightness is chosen: this property makes it possible to very quickly optimize the shape and the position of the optimum resonance for a selected brightness. Due to the high brightness sensibility of the human eye around the green range [ 450 to $650 \mathrm{~nm}$ ], an extremely narrow resonance must be used to balance blue or red colors with a same brightness.

In a second part, we have explained how to design a GWS to obtain a reflection spectrum with a single peak and a transmission close to 1 over the whole visible spectrum. Instead of directly using a simulation software and of scanning the different parameters to obtain saturated colors, we have explained how to quickly obtain the geometry and optimize such a structure by decorrelating the different geometric parameters. The first optimization step only requires a 1D optical mode solver and makes possible to size the filling factor of the PC and the thickness of every layer in order to maximize the amount of light transmitted across all the visible range. The PC is partially etched to obtain a residual layer as thin as possible to provide a guided mode with a moderate increase of the background reflection. Optimization with RCWA is particularly fast since the previously calculated thicknesses are accurate initial guesses, and computation for only few wavelengths is required. Last, increasing the period of the PC helps in positioning the Fano resonance peak at the right wavelength for color management: more generally, lateral size of the pattern controls the reflected spectrum properties.

Producing multicolor semi-transparent surfaces with a single nano-patterning step implies that the same thicknesses and partial etching depth must be considered for all the chosen colors. This condition greatly limits the maximum color saturation since the obtained solution corresponds to $R_{0}>7 \%$. For outdoor photovoltaic applications, this broadband reflection also reduces the optical transmission and so conversion efficiency. However, one could theoretically reduce this parasitic reflection using a double-layer ARC and materials with suitable dispersion curve such as different silicon oxynitrides. The main drawback of this solution is the narrowing of the optical filter bandpass that may also impact the conversion efficiency of a solar cell because of a high reflection in the infrared domain.

Last, to reduce the impact of the sun position and of the human being toward the patterned surface, one may consider more complex PCs as demonstrated in Ref. 16 or satinated front surfaces as proposed in Ref. 17.

\section{Acknowledgments}

The authors would like to thank the NanoLyon technological platform at INL for $\mathrm{TiO}_{2}$ layers processing and their optical measurement. 


\section{References}

1. K. Knop, "Rigorous diffraction theory for transmission phase gratings with deep rectangular grooves," J. Opt. Soc. Am. A 68(9), 1206-1210 (1978).

2. S. Sun et al., "All-dielectric full-color printing with $\mathrm{TiO}_{2}$ metasurfaces," ACS Nano 11(5), 4445-4452 (2017).

3. G. Quaranta et al., "Recent advances in resonant waveguide gratings," Laser Photonics Rev. 12(9), 1800017 (2018).

4. M. Amara et al., "Temperature and color management of silicon solar cells for building integrated photovoltaic," EPJ Photovoltaics 9, 1 (2018).

5. Committee on Colorimetry, "Quantitative data and methods for colorimetry," J. Opt. Soc. Am. 34, 633-688 (1944).

6. D. L. MacAdam, "Projective transformations of I. C. I. color specifications," J. Opt. Soc. Am. A 27(8), 294-299 (1937).

7. R. W. G. Hunt and M. R. Pointer, Measuring Colour, John Wiley \& Sons (2011).

8. O. Stenzel et al., "Observation of the waveguide resonance in a periodically patterned high refractive index broadband antireflection coating," Appl. Opt. 53(14), 3147-3156 (2014).

9. W. H. Press et al., Numerical Recipes: The Art of Scientific Computing, 3rd ed., Cambridge University Press (2007).

10. M. Moharam et al., "Formulation for stable and efficient implementation of the rigorous coupled-wave analysis of binary gratings," J. Opt. Soc. Am. A 12(5), 1068-1076 (1995).

11. P. Lalanne and M. Hutley, "Artificial media optical properties-subwavelength scale," Encycl. Opt. Engin. 1, 62-71 (2003).

12. L. A. Coldren, S. W. Corzine, and M. L. Mashanovitch, Diode Lasers And Photonic Integrated Circuits, 2nd ed., Vol. 218, John Wiley \& Sons (2012).

13. A. Snyder and J. Love, Optical Waveguide Theory, Science Paperbacks, Springer US (1983).

14. K. Kawano and T. Kitoh, Introduction to Optical Waveguide Analysis: Solving Maxwell's Equation and the Schrödinger Equation, John Wiley \& Sons (2004).

15. R. Orobtchouk et al., "High-efficiency light coupling in a submicrometric silicon-oninsulator waveguide," Appl. Opt. 39(31), 5773-5777 (2000).

16. C. Xu et al., "Design of full-k-space flat bands in photonic crystals beyond the tight-binding picture," Sci. Rep. 5, 18181 (2015).

17. N. Jolissaint et al., "Colored solar façades for buildings," Energy Procedia 122, 175-180 (2017).

18. D. Smith et al., "Handbook of optical constants of solids," Handbook of Optical Constants of Solids, Vol. 1, pp. 369-406, Academic Press, Inc., Orlando (1985).

Fabien Mandorlo received his MS degree in electronics from the University of Lyon and his engineer diploma from the École Centrale de Lyon, France, in 2005. Then, he conducted research both at CEA-LETI and INL (Lyon Institute of Nanotechnologies). In 2010, he joined the Silicon Photonics team at the Institut d'Electronique Fondamentale (IEF) to work on Si-Ge APD photodiodes for telecom applications. Since 2011, he has been a part of the photovotaic team of the INL.

Mohamed Amara is a CNRS researcher. He obtained his PhD in 2004 from the University of Limoges on the synthesis of $\mathrm{Si} / \mathrm{C} / \mathrm{N}$ nano-powders by laser pyrolysis. In 2008 , he joined CETHIL -UMR5008 after post-doctoral studies of one year at UNSW in order to develop research on thermal phenomena involving in solar cells and modules. In 2018, he joined the photovoltaic team at the Institute of Nanotechnology of Lyon.

Hai Son Nguyen has a $\mathrm{PhD}$ in physics (2011) for his works on single photon emissions of semiconductor quantum dots from École Normale Supérieure de Paris. From 2011 to 2014, he was a CNRS postdoc researcher, working on quantum fluid of light based on exciton-polaritons. Since 2014, he has been an associate professor at the École Centrale de Lyon and researcher at the Institut des Nanotechnologies de Lyon. He specializes in nanophotonics, condensed matter physics, and quantum optics. 
Arthur Charlety-Meano was an engineering student at INSA Lyon, a post-baccalaureate high school. He is now a PhD student at the Institute of Earth Sciences (ISTerre) to study landslides.

Ali Belarouci received his PhD from the University of Lyon, France, in 1999. He is currently a senior research scientist at the French National Research Agency (CNRS). His research interests include nanophotonics and plasmonics, physics of light-matter interaction at the nanoscale, nanoscale heat management, and energy harvesting.

Régis Orobtchouk received his PhD from the University of Paris XI, Paris, France, in 1996. In 1998, he joined the Institut des Nanotechnologies de Lyon Laboratory, INSA de Lyon, Université de Lyon, as an assistant professor. His main research interests are focused on silicon-based photonics for optical interconnect, telecommunication, and sensors applications. A part of his research is also related to the fabrication of periodic nanostructure by holographic method. 